\title{
Zur Managementforschung
}

\author{
Peter Conrad • Jochen Koch • Jörg Sydow
}

(C) Springer Fachmedien Wiesbaden 2016

Ziel der „Managementforschung“ (MF) ist es, einen Überblick über den aktuellen Stand und Ergebnisse der Forschung zu Managementproblemen zu geben; zugleich soll sie ein Diskussionsforum für neue Trends und Strömungen im gesamten Bereich des Managements sein. Die MF versteht sich als eine transdisziplinäre Fachzeitschrift für avancierte Fragen und kritische Analysen der Koordination und Steuerung komplexer sozialer Systeme, insbesondere von Organisationen, ihren Mitgliedern und Teilnehmern, aber auch von interorganisationalen Netzwerken, Communities oder Crowds. Sie steht in der Tradition eines verhaltens- und sozialwissenschaftlichen Paradigmas, ist aber grundsätzlich offen für alle Theorieströmungen, die einen Beitrag zur Lösung von Managementproblemen leisten. Die zur Begutachtung eingereichten Beiträge können sowohl rein konzeptioneller Art sein als auch über Ergebnisse empirischer Studien berichten. Die MF bietet neben den klassischen Aufsatzformaten zusätzlich auch eine Plattform für Beiträge in der Form kontroverser Essays, an die sich Diskussionen anschließen können.

Die MF ist am Institut für Management, dem heutigen Management-Department der Freien Universität Berlin entstanden und erscheint seit 1991 jährlich. Sie wurde zusammen mit Wolfgang H. Staehle $(\dagger)$ gegründet und viele Jahre von Georg Schreyögg zusammen mit Peter Conrad und Jörg Sydow herausgegeben. Seit 2012

P. Conrad

Hamburg, Deutschland

E-Mail: peter.conrad@hsu-hh.de

J. Koch $(\bowtie)$

Frankfurt (Oder), Deutschland

E-Mail: koch@europa-uni.de

J. Sydow

Berlin, Deutschland

E-Mail: joerg.sydow@fu-berlin.de 
(Band 22) wird die MF gemeinsam von Peter Conrad, Jochen Koch und Jörg Sydow herausgegeben. Der Schwerpunkt liegt nach wie vor auf innovativen Forschungsbeiträgen zu zentralen Gebieten des Managements. Neben anerkannten Fachvertretern und Fachvertreterinnen haben auch qualifizierte Nachwuchswissenschaftler/innen die Gelegenheit, zu aktuellen Fragen Stellung zu nehmen. Disziplinäre Offenheit ist Programm. Die Herausgeber werden bei der Akquisition, Begutachtung und Auswahl geeigneter Beiträge durch den Herausgeberbeirat unterstützt. Diesem gehören zurzeit an:

aus dem Bereich der Betriebswirtschaftslehre

- Prof. Dr. Albrecht Becker, Universität Innsbruck

- Prof. Dr. Peter Eberl, Universität Kassel

- Prof. Dr. Torsten J. Gerpott, Universität Duisburg-Essen

- Prof. Dr. Axel Haunschild, Universität Hannover

- Prof. Dr. Werner Hoffmann, Wirtschaftsuniversität Wien

- Prof. Dr. Dirk Holtbrügge, Universität Erlangen-Nürnberg

- Prof. Dr. Helmut Kasper, Wirtschaftsuniversität Wien

- Prof. Dr. Bernd Schauenberg, Universität Freiburg

- Prof. Dr. Frank Schirmer, TU Dresden

- Prof. Dr. Antoinette Weibel, Universität St. Gallen

- Prof. Dr. Jürgen Weibler, FernUniversität in Hagen

- Prof. Dr. Elke Weik, Universität Leicester

- Prof. Dr. Ingo Weller, LMU München

- Prof. Dr. Uta Wilkens, Universität Bochum

aus dem Bereich der Arbeits- und Organisationssoziologie bzw. -psychologie und der Politologie

- Prof. Dr. Jens Beckert, MPIfG Köln

- Prof. Dr. Ulrich Jürgens, Wissenschaftszentrum Berlin

- Prof. Dr. Friedemann Nerdinger, Universität Rostock

- Prof. Dr. Sigrid Quack, Universität Duisburg-Essen

Alle eingereichten und zur Begutachtung angenommenen Manuskripte durchlaufen einen doppelt-blinden Reviewprozess mit mindestens zwei Fachgutachterinnen bzw. Fachgutachtern. Darüber hinaus behalten sich die Herausgeber die Möglichkeit vor, spezielle Beiträge für die Managementforschung einzuladen und zu begutachten. In der Regel erfolgt die Begutachtung der Beiträge aus Sicht verschiedener Disziplinen. Auf der Grundlage der Gutachten wird über die Annahme sowie über Art und Umfang einer gewünschten Überarbeitung der Manuskripte entschieden. Jeder Band der Managementforschung ist somit das Ergebnis einer engen Kooperation zwischen Autoren, Beiräten, weiteren externen Gutachtern und den Herausgebern. Die MF akzeptiert ausschließlich Originalbeiträge.

Eine Einreichung von Beiträgen ist im Unterschied zu den Vorjahren nun jederzeit möglich. Mit Band 26 erscheint ein akzeptierter Beitrag bei Einreichung bis zum 30. Juni eines Jahres unmittelbar nach Annahme online (,online first“). Sämtliche Beiträge eines Jahres werden jährlich zusammengefasst publiziert. 


\section{Vorwort}

Alle im Band 26 der MF publizierten Beiträge wurden - dies nun zum ersten Mal in der Geschichte der Zeitschrift - bereits „online first“" veröffentlicht. Beim ersten und letzten Beitrag dieses Bandes handelt es sich - wie auch beim abschließenden Kommentar - um jeweils eingeladene Beiträge, die ein offenes Reviewverfahren durchlaufen haben. Thematisch und in der Gesamtschau betrachtet entfalten die Beiträge ein spannungsreiches Spektrum an aktuellen Forschungsfragen, das von der kritischen Analyse einer ökonomischen Fundierung der Managementfunktion „Führung“, über die Analyse des Verhältnisses von Individuum und Organisation, zu Fragen der Routinendynamik, der Employer-Attraktivität und der Unsicherheitswahrnehmung in interorganisationalen Netzwerken reicht und in einer steuerungstheoretischen Analyse von Zukunftspraktiken in Organisationen und einen diesen Ansatz kommentierenden Beitrag mündet.

Den Auftakt machen Jürgen Weibler \& Sigrid Endres, die in ihrem Aufsatz der Frage nachgehen, inwiefern das ökonomische Theoriegebäude in der Lage ist, einen Beitrag zum Verständnis von Führungsphänomenen zu leisten. Die Autoren kennzeichnen die sich abzeichnende Tendenz, auch personale Führung mittels etablierter ökonomischer Ansätze, wie etwa der Prinzipal-Agenten-Theorie, zu begreifen, als Leadership-Ökonomik und untersuchen auf der Basis eines umfassenden Reviews, den damit einhergehenden Erkenntnisgewinn. Ihre Bestandsaufnahme fällt insgesamt eher ernüchternd aus. Als einen Hauptgrund für diesen Zustand identifizieren die Autoren die der Leadership-Ökonomik immanenten limitierenden Annahmen hinsichtlich sozialer Interaktion und Entwicklung von Beziehungen.

Peter Preisendörfer adressiert mit seinem Beitrag ein gleichermaßen klassisches wie nach wie vor hoch aktuelles Thema: das Verhältnis von Individuum und Organisation. Dabei fokussiert er auf einen in diesem Zusammenhang selten in den Mittelpunkt gerückten Bereich, der sich nicht auf die organisationalen Binnenbeziehungen, sondern auf das Außenverhältnis von Organisationen bezieht. Der Autor argumentiert, dass es speziell in modernen, fortgeschrittenen Gesellschaften zu einem „Informalitätsdruck“ kommt und dass gleichermaßen öffentliche Organisationen wie auch Unternehmen zunehmend auch informale Beziehungen im Außenverhältnis etwa zu Bürgern oder zu Kunden - pflegen. Der Autor diskutiert diese Entwicklung vor dem Hintergrund des sich einstellenden Spannungsfeldes von Formalität und Informalität sowohl aus der Perspektive der Organisation wie aus Sicht des Individuums.

Im dritten Beitrag des Bandes geht Anja Danner-Schröder der Frage nach, wie der routinenbasierte Handlungsfluss in Organisationen angesichts interruptiver, eben diesen Fluss unterbrechender Ereignisse, besser verstanden und konzeptionalisiert werden kann. Auf der Basis einer ethnografischen Studie im Bereich des Katastrophenmanagements kontrastiert die Autorin zwei unterschiedliche Fälle mit jeweils ununterbrochenen und unterbrochenen routinebasierten Handlungsverläufen. Auf den gewonnenen Einsichten basierend argumentiert die Autorin dafür, dass die Akteure in Situationen unterbrochener Verläufe durch Wechsel des Handlungsmodus von Ausübung der Routine auf Reflexion der Routine praktisch zu einem ,perfomance repair“ kommen können. Interessant dabei ist insbesondere, dass die Autorin 
diesen Reflexionsmodus als Teil der Routine selbst - und dieser mithin immanent konzeptionalisiert.

Janina Merk \& Marion Büttgen untersuchen in ihrem Beitrag, inwieweit das arbeitgeberseitige Angebot von gesundheitsfördernden, die physische und psychische Leistungsfähigkeit der Belegschaft erhöhenden Maßnahmen, einen Effekt auf die wahrgenommene Arbeitgeberattraktivität hat. Die Autorinnen kommen auf der Basis ihrer Untersuchung mit etwas über 3000 Mitarbeiterinnen und Mitarbeitern aus deutschen Unternehmen zu der Erkenntnis, dass die wahrgenommene Verfügbarkeit solcher Maßnahmen alleine nicht ausreicht, um die Attraktivität eines Arbeitgebers zu erhöhen, sondern diese insgesamt von dem jeweiligen Arbeitgeberbranding abhängt.

Auf der Basis einer longitudinal angelegten Untersuchung eines SAP-Partnernetzwerks im Bereich Cloud Computing untersuchen Timo Braun \& Thomas Schmidt die Entwicklung dieses Verbundes mit Blick auf die prozessual sich veränderte Wahrnehmung von Unsicherheit. Mit der Frage, in welcher Beziehung Netzwerkentwicklung und Unsicherheitswahrnehmung durch die Netzwerkpartner stehen, eröffnen die Autoren eine bisher eher vernachlässigte Perspektive, die gleichermaßen für die Netzwerksteuerung als auch für Fragen der Unsicherheitsverarbeitung von Systemen relevant ist. Auf der Basis ihrer empirischen Befunde und der zeitlichen Entfaltung des Entwicklungsprozesses des Partnernetzwerkes argumentieren die Autoren, dass lediglich eine moderate Wahrnehmung von Unsicherheit zur Stabilisierung und Fortentwicklung des Netzwerkes beiträgt, wohingegen niedrige oder sehr hohe Unsicherheitswahrnehmung zur Netzwerkauflösung bzw. zur Verlangsamung der Netzwerkentwicklung führt.

Der letzte Beitrag dieses Bands 26 der MF befasst sich aus steuerungstheoretischer Sicht mit der Frage, in welcher Art und Weise Zukunft in Organisationen verund bearbeitet wird. Jochen Koch, Hannes Krämer, Andreas Reckwitz \& Matthias Wenzel schlagen dazu eine praxistheoretische Perspektive vor und argumentieren, dass damit eine systematische Grundlage geschaffen werden kann, Zukunft in Organisationen tatsächlich jenseits eines genuinen Planungsproblems zu begreifen. Insbesondere argumentieren die Autoren auch für ein empirisch offenes Verständnis des Umgangs mit Zukunft in Organisationen, um auf diese Weise die vielfältigen, heterogenen Formen der Zukunftsbearbeitung zu erfassen und Zukunft auf der Basis eines in der Gegenwart stattfindenden, performativen und situativen Handlungsvollzugs zu verstehen.

Abschließend kommentiert Jens Beckert diesen Beitrag insbesondere vor dem Hintergrund der Frage, inwiefern es den Autoren gelingen kann, mit einer praxistheoretischen Grundlegung von Zukunft in Organisationen zugleich ein Forschungsprogramm zu begründen. Der Kommentator sieht hierzu durchaus fruchtbare Ansätze, macht jedoch zugleich weiteren Bedarf an Konkretisierung aus.

Im Rahmen des doppelt-blinden Begutachtungsverfahrens wurden die Gutachten für die eingereichten Beiträge mit großer Sorgfalt und Engagement von den Mitgliedern des Herausgeberbeirats der MF erstellt. Darüber hinaus haben als Gutachterinnen und Gutachter für diesen Band mitgewirkt: 
- Prof. Dr. Daniel Geiger, Universität Hamburg

- Prof. Dr. Indre Maurer, Universität Göttingen

- Prof. Dr. Alfred Kieser, Zeppelin Universität und Universität Witten-Herdecke

- Prof. Dr. Arnold Windeler, TU Berlin

- Prof. Dr. Johannes Lehner, Johannes Keppler Universität Linz

Den Mitgliedern des Beirats und den externen Gutachterinnen und Gutachtern danken wir an dieser Stelle für ihre Unterstützung beim Erstellen dieses Bandes sehr herzlich.

Mit dem Erscheinen von Band 26 der MF geht erneut eine personelle Veränderung im editorischen Management einher. Natalie Senf, die unser Journal in den letzten zwei Jahren mit viel Umsicht administrativ gesteuert hat, wird nun von Dr. Matthias Wenzel als neuem „Managing Editor“ abgelöst. Wir danken Natalie Senf für die geleistete Arbeit und ihren tollen Einsatz für die MF - und damit für die Managementforschung - in der Phase eines für alle Beteiligten nicht immer leichten editorischen Umstellungsprozesses!

Hamburg, Frankfurt (Oder) und Berlin, im September 2016

Peter Conrad, Jochen Koch und Jörg Sydow 